\title{
Effects of Different Modes of Labor Analgesia on Neonatal Neurobehavior
}

\author{
Yu Han', Qiu Meng², Zhuojiu Du¹, Lianfang Chen'1, Xianmei Wei', Peijia Wei', Xiaohua Huang1, \\ Biyun Zhou1, Xiangli Feng1, Haiyan Lin ${ }^{1 *}$
}

${ }^{1}$ The Fourth People's Hospital of Haikou, Haikou, China

${ }^{2}$ Haikou People's Hospital, Haikou, China

Email: ^2252815531@qq.com

How to cite this paper: Han, Y., Meng, Q., Du, Z.J., Chen, L.F., Wei, X.M., Wei, P.J., Huang, X.H., Zhou, B.Y., Feng, X.L. and Lin, H.Y. (2021) Effects of Different Modes of Labor Analgesia on Neonatal Neurobehavior. Open Journal of Anesthesiology, 11, 369-377.

https://doi.org/10.4236/ojanes.2021.1112037

Received: November 29, 2021

Accepted: December 25, 2021

Published: December 28, 2021

Copyright $\odot 2021$ by author(s) and Scientific Research Publishing Inc. This work is licensed under the Creative Commons Attribution International License (CC BY 4.0).

http://creativecommons.org/licenses/by/4.0/

\begin{abstract}
The term "painless delivery" originated from foreign countries and has a history of more than 100 years. It is actually called "childbirth analgesia" in medicine. Labor analgesia, as its name implies, is the use of various methods to reduce or even eliminate the pain during labor. With the development of anesthesiology and pain, it has been widely used in foreign countries, especially in some developed countries in the West. The rate of labor analgesia in the United States is $>85 \%$, and even as high as $90 \%$ in Britain. The best childbirth analgesia should include side effect is small to puerpera and fetus, exact analgesic effect, quick effect, maintain time is long, can satisfy normal labor course; puerpera is awake, can cooperate childbirth and do not affect uterine contraction, and do not affect labor course progress. In 2000, WHO proposed that medical institutions should provide various labor analgesic services for parturient women to reduce labor pain as much as possible. Encourage the use of non-pharmaceutical analgesic techniques. In this paper, the effects of different modes of labor analgesia on neonates are described as follows.
\end{abstract}

\section{Keywords}

Painless Delivery, Labor Analgesia, The Newborn, Factors Nerve

\section{Preface}

The term "painless delivery" was originated from foreign countries and has a history of more than 100 years. It is actually called "childbirth analgesia" in medicine. Labor analgesia, as its name implies, is the use of various methods to reduce or even eliminate the pain during labor. With the development of anesthe- 
siology and pain, it has been widely used in foreign countries, especially in some developed countries in the West. The rate of labor analgesia in the United States is $>85 \%$, and even as high as $90 \%$ in Britain. However, there is no detailed statistical data in China, it is understood that the rate of labor analgesia in China is less than $1 \%$ in the early years. With the vigorous implementation of labor analgesia technology and concept in China in recent years, although the labor analgesia rate has been improved, even in developed cities such as Beijing, the labor analgesia rate is still less than $10 \%$. According to a survey released by the World Health Organization in 2010, the rate of cesarean section in China is as high as $46.2 \%$, ranking first in the world, and the proportion of cesarean section without surgical indication in China accounts for $11.7 \%$ of all cesarean sections [1]. In 2014, a national multi-center study on the indications of cesarean section suggested that "mother's requirement" ranked first, accounting for $24 \%$, followed by fetal distress, midscale cephalic pelvis, scar uterus, abnormal fetal position, etc. In CDMR, labor pain due to fear was as high as $19.75 \%$ [2]. A growing number of research shows that the high cesarean section rate and deformity development in our country is one important reason why women fear of labor pain [3], this abnormal rising cesarean delivery rate improves the safety of mothers and the survival rate of newborns, on the contrary, makes caesarean section maternal and neonatal casualties and increases the risk of postoperative complications [4] [5]. Reasonable application and implementation of labor analgesia in parturient is an effective means to reduce the rate of cesarean section. Theoretically, as long as labor pain can be alleviated, part of the cesarean section can be reduced, even if the application of non-drug analgesia technology can reduce the cesarean section rate by at least $5 \%$ or even more [6]. On the other hand, labor analgesia also has great social benefits and obvious economic benefits [7]. However, the popularization of labor analgesia is not enough at present, and the influence of labor analgesia on puerpera and newborns is still not comprehensive, which restricts the wide development of labor analgesia. Therefore, it is of great clinical and social significance to study and compare the effects of labor analgesia methods on mothers and children.

\section{Mechanism and Characteristics of Labor Pain}

Labor pain is a paroxysmal, progressively enhanced, blunt acute pain, mainly produced in the first and second stages of labor. It is important to understand the mechanism of labor pain, such as the peripheral mechanism and conduction pathway of labor pain, and the factors affecting the nature, duration and severity of labor pain.

\subsection{Mechanism of Labor Pain}

The first stage of labor refers to the stage from regular uterine contraction to complete expansion of the cervix, which is classified as latent Phase and active phase. At this point, the pain is in essence "visceral pain", this pain is often blunt pain 
and unclear positioning is mainly caused by two factors: spasmodic contraction of uterine smooth muscle and cervical dilation. When the uterus contracts, it causes uterine muscle ischemia, which causes the body to release bradykinin, 5-hydroxytryptamine, histamine and other mediators. At the same time, the dilation of the lower uterine segment and cervix can stimulate the mechanoreceptors, which are transmitted to the T10-L1 spinal cord through the lower abdominal nerves and then uploaded to the posterior central gyrus of the brain for perception [8]. During the incubation period of the first labor stage, intrauterine pressure reached $5.33 \mathrm{kPa}$ during contractions lasting $70 \mathrm{~s}$, and pain lagged contractions appeared in $20 \mathrm{~s}$ and lasted $30 \mathrm{~s}$. In the active period, uterine contractions when the intrauterine pressure reached $6.67 \mathrm{kpa}$, lasting $75 \mathrm{~s}$, pain hysteresis contractions appear $10 \mathrm{~s}$, lasting $40 \mathrm{~s}$. Into the second stage of labor, with the further expansion of the birth canal, the uterine body and its lower segment contraction is more intense, the pain is more obvious. During the second stage of labor, the intrauterine pressure reached $5.67 \mathrm{kp}-8.0 \mathrm{kpa}$, and the pain hysteretic contractions appeared for $5 \mathrm{~s}$ after the duration of $80 \mathrm{~s}$. In addition, the pressure on the pelvic cavity and perineum also increases gradually, becoming one of the main sources of pain [9]. The scholar thinks right now ache is "viscera are painful + body is painful". The generation mechanism of labor pain varies with the different stages of labor, which determines the differences in analgesic methods [6]. On the other hand, there are individual differences in the sensory nerves innervating the uterine body in the neural pathway of labor pain. Most of them are the SPINAL nerves of T11 or T12, but sometimes they also pass through T10, L1 and L2, while the sacral nerves S2, S3 and S4 innervate the lower segment of uterus and cervix. Labor pain generally manifests itself on the surface of these sensory nerves. During the first stage of labor, pain is located in the superficial areas dominated by T11 and T12. As labor progresses, the intensity and duration of contractions increase, and labor pain gradually presents as spasmodic sharp pain [10], and expands to the body surface dominated by T10 and L1. At this time, many women often complain of lumbago. However, after entering the active first stage of labor, pain is mainly concentrated in the sacral area, perineum and thigh. In addition to the changes of uterine contraction intensity, duration and type, physical factors, psychological factors, social factors, environmental factors will affect the subjective feeling of pain. The physical factors affecting labor pain include age, maternal physical conditions, cervical conditions, relative to the size of the fetus in the birth canal, etc. There is also a certain degree of interaction between various factors, generally young primiparas due to severe anxiety pain is more serious, dystocia, head basin is not weighed, fetal position is not correct, maternal unit height weight is heavier, fetal weight is heavier, labor pain is heavier.

\subsection{Adverse Effects of Labor Pain on Mothers and Children}

Labor pain can have serious adverse effects on mothers and children, leading to maternal hyperventilation, Hypoxemia, increased blood pressure, increased car- 
diac output, inhibition of gastrointestinal peristalsis, uncoordinated contractions, and strong physical stress; it can cause fetal distress in utero and increase neonatal complications. Adverse effects of labor pain on the mother: labor pain on the maternal respiratory system, cardiovascular system, digestive system and endocrine system are affected. Labor pain during contractions can strongly stimulate breathing, deep and fast breathing significantly increased the maternal alveolar ventilation, the formation of hyperventilation, $\mathrm{PaCO}_{2}$ significantly reduced, blood $\mathrm{pH}$ increased to form respiratory alkalosis. In the intermittent period of contractions, the disappearance of respiratory stimulation of contractions, coupled with hypocapnia, makes maternal breathing shallow and slow, so transient hypoxemia can occur. $\mathrm{PaO}_{2}$ is usually reduced by $25 \%$ to $30 \%$, which will affect the fetus when it is as low as $70 \mathrm{mmHg}$. In addition, uterine contractions will squeeze out $250-300 \mathrm{ml}$ blood from the uterus to the systemic circulation, with each contraction of the heart will increase the volume of blood, can be compared to the average increase before delivery of $40 \%-50 \%$, some maternal, even in uterine contractions can increase $120 \%$ - 130\%. In addition, pain activates the sympathetic-adrenal-aldosterone system, raising blood pressure. These conditions increase the work of the heart and are well tolerated by healthy pregnant women, but can lead to cardiac insufficiency and acute heart failure in pregnant women with heart disease. In addition, labor pains excite the sympathetic adrenal aldosterone system, which creates intense stress in the body and increases blood levels of adrenaline, norepinephrine, and cortisol several times over. Labor pain can also increase the secretion of gastrin and gastric acid, inhibit gastrointestinal peristalsis, gastrointestinal empties. Severe pain is also a high risk factor for postpartum depression [11]. In the process of labor, good analgesia can eliminate labor pain caused by hyperventilation, to avoid transient hypoxia; In particular, COMPARED with epidural anesthesia, CSEA can significantly reduce the amplitude of blood pressure, heart rate and heart index, effectively reducing the burden of the heart [12], especially for pregnant women with heart disease, pregnancy-induced hypertension and pulmonary hypertension. It can obviously reduce the oxygen consumption of the body, alleviate metabolic acidosis and reduce the stress of the body. It can relieve gastrointestinal function inhibition caused by labor pain, and labor analgesia itself does not affect gastrointestinal function. Labor analgesia labor pains for uterine contractions and delivery also have an impact. Labor pain affects uterine contractions through increased catecholamines, increased norepinephrine enhances contractions, and increased adrenaline and cortisol inhibit contractions. Labor pain can lead to uncoordinated contractions, which are characterized by inadequate intensity, increased frequency, or spasmodic contractions. Good analgesia can effectively inhibit excessive sympathetic reactions and eliminate uncoordinated uterine contractions. It is also important to eliminate the hypoperfusion state of the uterus by returning to normal contractions in favor of fetal oxygenation. Adverse effects of labor pain on the fetus: First of all, when the uterus has regular contractions, uterine blood flow is sig- 
nificantly reduced during contractions, so that the gas exchange with the fetus is significantly reduced. Next, excessive ventilation during labor can cause respiratory alkalosis. The oxygen dissociation curve shifts to the left as a result of respiratory alkalosis, reducing the release of oxygen to the fetus. In addition, in the period between contractions of the mother's respiratory depression, resulting in hypoxemia also make fetal hypoxia further aggravated. During normal labor, the fetus can withstand a short period of maternal hypoxia with good oxygen reserves in its own blood circulation. The combination of multiple adverse factors during labor or the impact of the mother's own disease or the poor tolerance of the fetus itself can increase the incidence of neonatal complications and mortality. Good labor analgesia, especially epidural analgesia, is beneficial to the fetus, especially at high risk. Although epidural analgesia can affect blood pressure, the overall result is an increase in placental blood flow of $77 \%-100 \%$, especially beneficial for pregnant women with preeclampsia, hypertension and diabetes. The results of this study showed that VAS score in NA group increased gradually with the progress of labor, and VAS score reached the highest value and pain was the most intense after uterine orificium opening. The SCORES of THE TENS group at $2 \mathrm{~h}$ after analgesia were the lowest, and the analgesic effect of the first labor stage was better than that of the second labor stage. The VAS score of CSEA group during the second labor stage was slightly higher than that at $1 \mathrm{~h}$ and $2 \mathrm{~h}$ after analgesia, but the VAS score was $3.6 \pm 1.1$, indicating mild to moderate pain and satisfactory analgesic effect.

\section{Effect of Labor Analgesia on Mother and Child}

\subsection{Effects of Analgesics or Anesthetics on Mothers and Children}

Anesthetics and anesthetics analgesics have different degrees of central inhibition, the main side effects are: 1) affect maternal respiration and produce hypercapnia and hypoxemia; 2) change maternal cardiovascular and hemodynamic state, thus affecting the uterine placental circulation; 3) affect uterine contraction; 4) the second stage of labor affects maternal force. Therefore, the key to rational drug use is to grasp the timing of drug use and understand the characteristics of drugs. Narcotic drugs and narcotic analgesics can have direct effects on the fetus, including cardiotoxicity and respiratory depression, or through the influence of maternal hemodynamics to alter the irrigation of uterine placenta $b$ lood flow, indirect effects on the fetus, resulting in fetal distress or neonatal acidosis.

\subsection{Effects of Inhaled Analgesia on Mother and Child}

The main danger of inhaled analgesia is the sudden inhalation of an overdose of anesthetics, resulting in the appearance of protective reflexes. Vomiting or asymptomatic regurgitant symptoms may appear at any time, resulting in respiratory obstruction and aspiration pneumonia. At present, nitrous oxide (laughing gas) inhalation labor analgesia is widely used at home and abroad, mostly inhaling 50\% 
nitrous oxide and $50 \%$ oxygen mixture. Inhaling nitrous oxide lasts too long, can cause maternal consciousness to disappear, and appear restless excitement, can make palace shrinkage weak; Prolonged ( $>50 \mathrm{~h}$ ) inhalation of nitrous oxide may have adverse effects on myelodysplasia, and its diffuse hypoxia effect may be harmful to the myocardium. Therefore, it is advisable to use with caution in patients with cardiopulmonary insufficiency, hematologic diseases and other medical complications and obstetric complications such as pregnancy induced hypertension. All inhaled anesthetics can act on the fetus through placenta, and the degree of inhibition to the fetus is related to the mass fraction of drugs in the mother's alveoli, lung ventilation and cardiac output. Maternal alveolar drug mass fraction is high, blood drug mass fraction is high, lasting for a long time, and has a great influence on the fetus. Clinically, inhaled anesthetics by mass fraction of linolenic acid are commonly used: $0.25 \%-0.8 \%$ halothane, $0.5 \%$ enflurane, $0.5 \%$ isoflurane, $3 \%$ desflurane, and 1:1 mixture of oxygen and nitrous gas. Satisfactory analgesic effect can be obtained by using this mass fraction. At this point, even if inhaled for a long time, there will be no significant inhibition of the fetus. Analgesic mass fraction of nitrous oxide can cause maternal and fetal hypoxemia, so often the use of subanesthesia mass fraction of nitrous oxide (33\% $50 \%)$ can have no effect on uterine contractions, when the concentration exceeds $75 \%$ to cause maternal blood oxygen deficiency, then uterine contraction is weak.

\subsection{Influence of Epidural Block on Mother and Child}

\subsubsection{Influence on Uterine Contraction}

Newton et al. [13] compared 62 women who used epidural analgesia with those who did not, and the results showed that the use of oxytocin increased in epidural labor analgesia group, and the rate of uterine expansion was significantly lower than that of non-epidural analgesia group $(1.5-5.6 \mathrm{~cm} / \mathrm{h}, \mathrm{P}<0.01)$. A retrospective study by Howell [14] showed that epidural analgesia prolonged the first and second stages of labor. Alexauder [15] believed that epidural block analgesia reduced uterine contraction activity and prolonged the first and second stages of labor in the process of labor enhancement with oxytocin, and epidural block did not affect uterine contraction. Chen et al. [16] showed that epidural block analgesia at the beginning of labor pain did not prolong labor, had no adverse effects on mothers and children, and did not affect the outcome of labor. It is believed that epidural analgesia block can coordinate the contractions and dilate the cervix. Correct use of abdominal pressure in semi-decubitus position after full cervix opening can overcome the adverse effects of epidural block analgesia on the second labor stage and have no adverse effects on the fetus. A large number of studies have shown that epidural injection of anesthetic analgesics and low-concentration local anesthetics can quickly and effectively produce analgesic effect, reduce the dosage of local anesthetics and block the motor nerve, and have a slight effect on the contraction, so it is more and more widely used. 


\subsubsection{Influence on Cesarean Section Rate}

Data suggest that epidural block during labor may increase the rate of cesarean section. Lieberman [17] estimated the relationship between epidural block and cesarean section through a retrospective investigation of 1733 primiparas with low risk pregnancy, cephalopod and single pregnancy, and the results showed that: The rate of cesarean section was $17 \%$ (168/991) in patients with epidural analgesia, and $4 \%(30 / 140)$ in patients without epidural analgesia. Logistic regression analysis showed that the risk of cesarean section in patients with epidural analgesia was 3.7 times higher than that in patients without epidural analgesia. However, Nagetie et al. [18] and Gambling et al. [19] reported that spinal canal block did not increase the cesarean section rate and dystocia rate of normal women and had no adverse effects on the fetus. Fung [20] reported that continuous epidural block analgesia did not increase the cesarean section rate, but reduced the cesarean section rate of primiparas. In a retrospective study, Hess et al. [21] used multivariate analysis and found that in epidural analgesia, the rate of cesarean section in women with more than 3 additional doses was 2.3 times that in women with less than 2 additional doses. Lidocaine concentration was not associated with delivery outcome. The reason may be that the puerpera in cesarean section has more severe labor pain and the degree of labor pain may be a confounding factor in the study of epidural analgesia and labor outcome.

\subsubsection{Influence on Placental Blood Flow}

Hypotension is a recognized adverse reaction of local anesthesia including epidural analgesia, subarachnoid analgesia or combined analgesia, but the effect on hemodynamics is not obvious when the local anesthetic concentration is reduced to a certain level. However, the effect on placental blood flow is sometimes not parallel to the change of blood pressure, and even in the absence of hypotension, epidural analgesia can redistribute shadow uterine blood flow [22]. Norris et al. [23] found that intraspinal block could slightly increase neonatal umbilical blood $\mathrm{PCO}_{2}$ after $5 \mathrm{~cm}$ of cervical opening. However, there are many reports that local block can improve placental blood flow.

\section{Conclusion}

With the improvement of people's economic and cultural living standards, people's concept has changed, and puerpera put forward higher requirements for doctors. The development of medical science has led to the transformation of the medical model. People have paid more attention to the influence of social and psychological factors on the process of childbirth. Clinical use of psychological intervention is helpful to relieve the pain in the process of puerpera childbirth. The medical personnel in the whole process of delivery of maternal give spiritual encouragement, psychological comfort, physical support, and help eliminate fear, anxiety, tension and maternal loneliness, so that make the process of delivery more smoothly, improve the quality of obstetric delivery and shorten the labor, reduce postpartum haemorrhage amount. The rate of cesarean section and neonatal asphyxia was reduced, 
so that the maternal delivery achieved satisfactory results. It is believed that the application of doula analgesia will promote the improvement of delivery level and make it possible for puerpera to give birth to a healthy baby safely and smoothly in a painless state.

\section{Foundation Project}

The paper is supported by Hainan Natural Science Foundation (Project No. 820MS166).

\section{Conflicts of Interest}

The authors declare no conflicts of interest regarding the publication of this paper.

\section{References}

[1] Lumbiganon, P., Laopaiboon, M., Gülmezoglu, A.M., Souza, J.P., Taneepanichskul, S., Ruyan, P., et al. (2010) Method of Delivery and Pregnancy Outcomes in Asia: The WTO Global Survey on Maternal and Perinatal Health 2007-2008. Lancet, 375, 490-499. https://doi.org/10.1016/S0140-6736(09)61870-5

[2] Hou, L., Li, G., Zhang, W., et al. (2014) A Study on the Prevalence of Cesarean Section in China. Chinese Journal of Obstetrics and Gynecology, 49, 729-735.

[3] Hao, F., Zhang, Y., Xiao, B., et al. (2012) Analysis of Non-Medical Indication Cesarean Section in Sichuan Province. Practical Gynecology and Obstetrics, 28, 187-190.

[4] Zhang, W. (2011) Current Situation and Thinking of Cesarean Section in China. Journal of Practical Obstetrics and Gynecology, 27, 161-163.

[5] Chen, X. and Pan, Y. (2012) The Current Situation and Thinking of Cesarean Section in China. Medical Review, 18, 3003-3006.

[6] Xu, M.J. (2012) Hot Issues Related to Vaginal Delivery Analgesia. Chinese Journal of Practical Gynecology and Obstetrics, 28, 110-115.

[7] Hu, L.Q., Flood, P., Li, Y., Tao, W., Zhao, P., Xia, Y., et al. (2016) No Pain Labor \& Delivery: A Global Health Initiative's Impact on Clinical Outcomes in China. Anesthesia \& Analgesia, 122, 1931-1938. https://doi.org/10.1213/ANE.0000000000001328

[8] Shol, H., Paul, N. and Belfer, I.B. (2014) Labor Pain Mechanisms. International Anesthesiology Clinics, 52, 1-17. https://doi.org/10.1097/AIA.0000000000000019

[9] Palanisamy, A., Mitani, A.A. and Tsen, L.C. (2011) General Anesthesia for Cesarean Delivery at a Tertiary Care Hospital from 2000 to 2005: A Retrospective Analysis and 10-Year Update. International Journal of Obstetric Anesthesia, 20, 10-16. https://doi.org/10.1016/j.ijoa.2010.07.002

[10] Zhao, X., Yin, J., Li, H., et al. (2015) Analgesic Mechanism and Research Progress of Percutaneous Nerve Electrical Stimulation. Journal of Practical Medicine, 31, 34803482.

[11] Pettersson, F.D., Hellgren, C., Nyberg, F., Åkerud, H. and Sundström-Poromaa, I. (2016) Depressed Mood, Anxiety, and the Use of Labor Analgesia. Archives of Women's Mental Health, 19, 11-16. https://doi.org/10.1007/s00737-015-0572-6

[12] Yacoubian, S., Oxford, C. and Kodali, B. (2017) Changes in Cardiac Index during Labour Analgesia: A Double-Blind Randomized Controlled Trial of Epidural versus Combined Spinal Epidural Analgesia-A Preliminary Study. Indian Journal of Anaes- 
thesia, 61, 295-301.

[13] Newton, E.R., Barbary, C. and Bennett, B.L. (1995) Epidural Analyesia and Uterine Function. Obstetrics \& Gynecology, 85, 749-755. https://doi.org/10.1016/0029-7844(95)00046-T

[14] Howell, C.-J. (2000) Epidural versus Non-Epidural Analgesia for Pain Relief in Labour. Cochrane Database of Systematic Reviews, 3, 1-6. https://doi.org/10.1002/14651858.CD000331

[15] Alexander, J.M., Lucas, M.J., Ramin, S.M., McIntire, D.D. and Leveno, K.J., (1998) The Course of Labor with and without Epidural Analgesia. American Journal of Obstetrics \& Gynecology, 178, 516-520.

https://doi.org/10.1016/S0002-9378(98)70431-4

[16] Chen, L.-K., Hsu, H.-W., Lin, C.-J., Huang, C.H., Tsai, S.K., Lee, C.N., et al. (2000) Effects of Epidural Fentanyl on Labor Pain during the Early Period of the First Stage of Induced Labor in Nulliparous Women. Journal of the Formosan Medical Association, 99, 549-553.

[17] Lieberman, E., Lang, J.M., Cohen, A., D’Agostino, J.R., Datta, S., Datta, S., et al. (1996) Assosiation of Epidural Analgesia with Cesarean. Delivery in Nulliporas. Obstetrics \& Gynecology, 88, 993-1000. https://doi.org/10.1016/S0029-7844(96)00359-6

[18] Nageotte, M.P., Larson, D., Rumney, P.J., Sidhu, M. and Hollenbach, K. (1997) Epidural Analgesia Compared with Combined Spinal-Epidural Analgesia during Laboring Nulliparous Women. The New England Journal of Medicine, 337, 1715-1719. https://doi.org/10.1056/NEJM199712113372402

[19] Cambling, D.R., Shama, S.K., Ramin, S.M., Lucas, M.J., Leveno, K.J., Wiley, J., et al. (1998) A Randomized Study of Combined Soinal-Epidural Analgesia versus Intravenous Meperidine during Labor: Impact on Cesarean Delivery Rate. Anesthsiology, 89, 1336-1344. https://doi.org/10.1097/00000542-199812000-00010

[20] Fung, B.-K. (2000) Continuous Epidural Analgesia for Painless Labor Does Not Increase the Incidence of Cesarean Delivery. Acta Anaesthesiologica Sinica, 38, 79-84.

[21] Hess, P.-E., Pratt, S.-D. and Soni, A.-K. (2000) An Association between Severe Labor Pain and Cesarean Delivery. Anesthesia \& Analgesia, 90, 881-886. https://doi.org/10.1213/00000539-200004000-00020

[22] Manninen, T., Aantaa, R. and Salonen, M. (2000) A Comparison of the Hemodynamic Effects of Paracervical Block and Epidural Anesthesia for labor Analgesia. Acta Anaesthesiologica Scandinavica, 44, 441-445. https://doi.org/10.1034/j.1399-6576.2000.440414.x

[23] Norris, M.C., Fogel, S.T. and Conway-Long, C. (2001) Combined Spinal-Epidural versus Epidural Labor Analgesia. Anesthesiology, 95, 913-920. https://doi.org/10.1097/00000542-200110000-00020 